\title{
DIE STAATSANGEHOORIGKEITSREGELUNGEN IN AUSTRALIEN UND DER SÜDSEE
}

\author{
Von Hellmuth Hecker
}

Nachdem im ersten Heft dieser Zeitschrift eine Übersicht über das Staatsangehörigkeitsrecht der damals 40 Staaten Afrikas gegeben worden war, folgt hier eine entsprechende Zusammenstellung für den fünften Kontinent. Dabei ist zu berücksichtigen, daß die Übersicht für Afrika weitgehend auf in der Sammlung Geltender Staatsangehörigkeits-Gesetze (SGS) erschienene Bände verweisen konnte, während für Australien noch kein Band dieser Reihe erschienen ist. Daher müssen hier alle Vorschriften primär nachgewiesen werden. Eine zweite Besonderheit der hier vorliegenden Ubersicht liegt darin, daß sie sich nicht nur, wie beim Schwarzen Kontinent, auf Staaten beschränkt, sondern auch das Staatsangehörigkeitsrecht der zahlreich abgestuften Formen abhängiger Gebiete einbezieht. Da gerade im Bereich der Südsee besonders vielfältig gestaltete Gebietseinheiten vorliegen, erschien es zweckmäßig, diese ohne Rücksicht auf ihre formelle staatsrechtliche Situation alphabetisch geordnet vorzulegen. Dabei ergeben sich 22 solcher Gebietseinheiten, die hier zu berücksichtigen sind. Es handelt sich dabei um 4 unabhängige Staaten (Australien, Neuseeland, West-Samoa und Nauru) und 18 abhängige Gebiete.

Von den unabhängigen Staaten sind die ersten drei Monarchien innerhalb des Commonwealth, Nauru dagegen eine Republik, die allerdings auch zum Commonwealth gehört. Australien ist ein Bundesstaat, dessen diverse Gliedstaaten früher auch eigene Staatsangehörigkeitsvorschriften besaßen. Neuseeland war 1852-1876 ebenfalls bundesstaatlich organisiert, gab diese Struktur aber wieder auf. Bei WestSamoa und Nauru stellt sich erstmalig das Problem der Staatlichkeit kleiner InselGebiete. Beide Gebiete sind zu klein, um beispielsweise eigene Auslandsvertretungen zu unterhalten oder sich zu schützen. Praktisch ruhen Außenpolitik und Verteidigung sowie zahlreiche Verwaltungsfunktionen weiterhin bei den bisherigen Mächten, so daß sie materiell eher Protektoraten gleichen. Die abhängigen Gebiete gliedern sich wie folgt:

a) 2 Kondominien: Neue Hebriden (Frankreich, Großbritannien) und Canton/Enderbury (Großbritannien, USA). Beide Gebiete sind keine Staaten, ihre Eingeborenen - solche gibt es aber nur bei den Neuen Hebriden - besitzen aber doch nach innen ihre eigene Gebietszugehörigkeit, während sie nach außen Schutzgenossen der Kondominanten sind, nicht aber deren Staatsangehörige.

Es ist bemerkenswert, daß in der Südsee die Form des Kondominiums anscheinend recht sinnvoll war. Es gab früher noch zwei weitere Kondominien, und zwar jeweils von sogar 3 Staaten: Samoa (Deutschland, USA, Großbritannien) und Nauru (Großbritannien, Australien, Neuseeland).

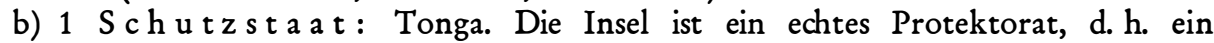
Gebilde, das eine Eigenstaatlichkeit mit eigener Staatsangehörigkeit besaß und sie in den Außenbeziehungen an den Protektor teilweise delegierte.

c) 1 a s soziierter St a a t: Die Cook-Inseln. Sie sind seit 1965 mit Neuseeland assoziierter Staat, haben aber keine eigene Staatsangehörigkeit.

d) 2 Treuhandgebie te : Die ehemals deutschen Kolonien Neuguinea und die Pazifischen Inseln von Mikronesien. Früher gehörten auch die inzwischen unabhängigen Inseln von Nauru und West-Samoa zu dieser Kategorie. Neuguinea ist praktisch von Australien eingegliedert; Mikronesien wurde nach dem japanischen Mandat dann den USA übertragen. Letzteres ist das einzige Treuhandgebiet, für das ausdrücklich eine Staatsangehörigkeitsbestimmung vorliegt (Artikel 11 des Treuhandvertrages).

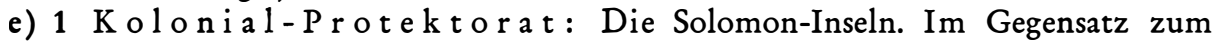
„Protected State" heißen die Kolonialprotektorate in der britischen Terminologie 
„Protectorate“. Sie besaßen keine organisierte Staatsgewalt, sondern nur eine Häuptlingsherrschaft. Das frühere französische Protektorat Wallis/Futuna bat 1959 um Annexion als Kolonie (Úberseegebiet). Das ehemalige britische Protektorat der Gilbert/Ellice-Inseln wurde 1916 Kolonie; ebenfalls waren drei heute neuseeländische Inselgebiete vorher britische Kolonialprotektorate.

f) $7 \mathrm{~K}$ ol o nie n : Zwei englische (Gilbert/Ellice, Fiji), drei französische (Polynesien, Neukaledonien, Wallis/Futuna) sowie zwei amerikanische (Guam und OstSamoa). Eigene Staaten, die annektiert wurden, waren davon Fiji und Tahiti (Polynesien), während die übrigen drei noch nicht eine verfaßte Staatlichkeit erreicht hatten. Von den genannten besitzen Fiji und Ost-Samoa eine eigene Verfassung.

h) 1 Gliedsta at: Hawaii. Das Königreich wurde 1893 Republik, 1898 amerikanisch, ist seit 1900 als US-Territorium verfaßt, 1959 als Staat der USA.

g) 3 A u Bengebiete: Niue, Tokelau und Papua. Zu Neuseeland gehören außer den Cook-Inseln noch zwei Inselgebiete mit gewisser Sonderstellung (als Art Region), ohne daß diese für die Staatsangehörigkeit Bedeutung hätte (Niue, Tokelau). Diese Inseln waren früher britische Kolonialprotektorate gewesen, die dann annektiert wurden. Von den australischen Außenterritorien besitzen die meisten keinerlei Sonderstellung, ausgenommen das Territorium Papua, das vor der Annexion auch Staatsangehörigkeitsvorschriften besaß. Die hier genannten drei Außengebiete sind von denjenigen der USA dadurch unterschieden, daß letztere mehr Kolonien ähneln und daher oben unter dieser Kategorie aufgeführt sind.

Die Verschiedenheit der abhängigen Gebiete bedingt naturgemäß auch eine Verschiedenheit der staatsangehörigkeitsrechtlichen Regelungen, die von einer echten Staatsangehörigkeit (Schutzstaat) über Schutzgenosseneigenschaft (Protektorate, Treuhandgebiete, Kondominien) zum Verlust einer eigenen Staatsangehörigkeit (Kolonien, Außengebiete, Gliedstaat) führen, wobei es bei letzteren aber doch noch gewisse Sonderrechte gibt, wie lokal begrenzte Einbürgerung in der britischen oder geminderte Staatsangehörigkeit in den amerikanischen Kolonien. Es zeigt sich dabei eine Tendenz, daß weit vom Mutterland enfernte Gebiete jedenfalls dann eine Entwicklung in Richtung auf eine Gebietszugehörigkeit nehmen, falls nicht das Mutterland so streng zentralistisch organisiert ist wie Frankreich. Die Verfassungslage des Mutterlandes wirkt sich eben immer auch auf die Art der Herrschaftsordnung in den abhängigen Gebieten aus.

Die Mächte, die in der Südsee staatliche Funktionen ausübten, sind nur wenige, nämlich England, Frankreich, Amerika, Deutschland, Japan. Holland kam über Niederländisch-Neuguinea, das hier wegen der Zugehörigkeit zu Indonesien außen vor blieb, nie hinaus. Und Spanien beanspruchte zwar von den Philippinen aus Mikronesien, richtete aber nie eine effektive Rechtsordung auf. Auch von Japan, das 1914-1945 Mikronesien als Mandat besaß, sind keine Staatsangehörigkeitsregelungen bekannt. So bleiben die oben genannten vier Mächte. Von ihnen schied Deutschland mit seinem nicht unerheblichen Kolonialbesitz (Samoa, Neuguinea, Nauru, Mikronesien) aus, bevor es die Schutzangehörigkeit der Kolonialuntertanen der Südsee gesondert geregelt hatte - die einzige Rechtsvorschrift vom 1. Juli 1900 definiert nur die Eingeborenen in Samoa. Gegenwärtig sind die 18 abhängigen Gebiete auf die drei Westmächte (Frankreich, USA, Großbritannien samt Australien und Neuseeland) verteilt, deren Rechtsordnung ihnen den Stempel aufprägte.

Eine eigene Staatlichkeit hat es vor der Einflußnahme der Europäer nur in Tahiti (heute Französisch-Polynesien), Hawaii und Tonga gegeben, während Samoa, Fiji und die Cook-Inseln erst mit europäischer Hilfe eine Art Verwaltung errichteten. Ob letztere drei Inselgruppen auch die Staatsangehörigkeit regelten, konnte nicht festgestellt werden, es scheint aber nicht der Fall gewesen $\mathrm{zu}$ sein ${ }^{1}$. Auch von

1 Der Court of Appeal in Neuseeland 2. B. verneinte am 6. 3. 1884 die Frage, ob es in Samoa ein 
einem Staatsangehörigkeitsgesetz Tahitis ist nichts bekannt. Dagegen erließen Hawaii und Tonga eigene Staatsangehörigkeitsvorschriften und eigene Einbürgerungsgesetze. Die erste Staatsangehörigkeitsbestimmung eines Südseestaates dürfte in der Verfassung Hawaiis von $1852 \mathrm{zu}$ finden sein, die erste Staatsanghörigkeitsvorschrift eines europäischen Staates in diesem Raum ist ein Einbürgerungsgesetz von Neusüdwales von 1828.

In der Literatur werden einzelne Gebiete dieses Raumes in ihrer politischen und verfassungsrechtlichen Entwicklung zwar behandelt, dagegen fehlt es noch an einem Werk über die Verfassungsgeschichte des gesamten Südsee-Raumes. Immerhin behandelt das Werk von Morell („Britain in the Pacific Islands“, Oxford 1960, 454 S.) bereits den wichtigsten Initiator der Erschließung des Südseeraumes, nämlich Großbritannien. Das Buch zeigt gut die ganze Buntheit der politischen Geschichte dieses weiten Raumes, die außer der Meuterei auf der Bounty (Gründung der Gemeinschaft von Pitcairn), der Wirtschaftsordnung der Godeffroys aus Hamburg auf Samoa noch so manche Abenteuer $\mathrm{zu}$ verzeichnen hat, nicht zuletzt die dramatischen Spannungen zwischen den evangelischen und den katholischen Missionaren als Vorboten der Herrschaft Großbritanniens und Frankreichs.

Der moderne Independismus als Gegenbewegung gegen den Kolonialismus hat im Südsee-Raum nach der Aufdrängung der Unabhängigkeit für Nauru noch ein weites Feld. Die Tausende von Inseln, die vor der Beeinflussung durch die Organisationsfähigkeit der Europäer meist je ein „Reich“ für sich bildeten, mögen, wenn das Beispiel Nauru Schule macht, irgendwann wieder in einem ähnlich individualistischen Zustand sein wie vor 100 Jahren: Eine Insel - ein Staat - ein Staatsangehörigkeitsgesetz.

\section{AUSTRALIEN}

A. Bundesrecht (Acts)

1. Australasian Naturalization Act (Federal) Nr. 1

Englischer Text: State Papers, Bd. 89, S. 461

2. Bundesverfassung (Brit. Gesetz 63/4 Vict. c. 12)

9.7. 1900

StAB: Sect. 51 (Ziff. 19), Bundeszuständigkeit für Einbürgerung von Ausländern

Texte: siehe Verfassungsregister, Bd. 4, S. 35

3. Naturalization Act, Nr. 11

Amtlicher Text: Commonwealth Acts, Bd. 2, S. 91

Englischer Text: Report US vom 20. 12. 1906, S. 362

State Papers, Bd. 96, S. 825

4. Naturalization Amd. Act, Nr. 25

20.9. 1917

Andert Sect. 3, 4, 6, 7, 10, 12

Englischer Text: State Papers, Bd. 111, S. 213

5. Nationality Act, Nr. 48

In Kraft: 1. 1. 1921 (Gazette vom 6. 1. 1921)

Amtlicher Text: Commonwealth Acts, Bd. 18, S. 139

Commonwealth Acts 1935, S. 99

Englischer Text: Parry, S. 533

Flournoy, S. 88 (Fass. 1925)

State Papers, Bd. 113, S. 259

Französische Übersetzung: Bourbousson, S. 254 (Fass. 1925)

6. Nationality Act, Nr. 24

18. 10.1922

Aindert Sect. 5, 7, 8, 11, 12, 14, 15, 29, 34; fügt ein Sect. 5 A

Amtlicher Text: Commonwealth Acts, Bd. 20, S. 116

Englischer Text: State Papers, Bd. 116, S. 281

Einbürgerungsgesetz gegeben habe und erkannte Samoa auch nicht als Staat an (NZLR 1884 I, S. 160-244). 
7. Nationality Act, Nr. 10

31. 8.1925

Ändert Sect. 5, 6; fügt ein Sect. 32 A

Amtlicher Text: Commonwealth Acts, Bd. 23, S. 13

Englischer Text: State Papers, Bd. 121, S. 337

8. Nationality Act, Nr. 9

29. 5.1930

Betr. Verfahren

Englischer Text: State Papers, Bd. 137, S. 75

9. Nationality Act, Nr. 62

In Kraft: 1. 4. 1937

Ändert Sect. 5, 17, 18, 34; fügt ein Sect. 18 A; ersetzt überall "Gen. gouv.“ durch „Minister“

Englischer Text: State Papers, Bd. 140, S. 127

10. Nationality Act, Nr. 9

18. 4.1946

In Kraft: 7. 11. 1946

Ändert Sect. 1 (Titel); fügt ein Sect. 18 B

11. Nationality Act (Nr. 2), Nr. 28

13. 8.1946

Erstreckung des StAG auf Neuguinea durch Einführung einer Sect. 5 B;

ändert Sect. 1 (Titel)

12. Nationality and Citizenship Act, Nr. 83

21. 12.1948

In Kraft: 26. 1. 1949 (Gazette 1949, S. 197)

Dazu Regulations von 1949, 1956 und 1960 je mit Änderungen (s. u.)

Amtlicher Text: Commonwealth Acts 1950, S. 2714

Englischer Text: Parry, S. 557

LCN, S. 13

State Papers, Bd. 151, S. 610

Bettoni, Bd. 1, S. 115 (Auszug)

Adriaansen, Af, S. 1

Holländische Übersetzung: Adriaansen, Af, S. 15

13. Nationality and Citizenship (Burmese) Act, Nr. 12

1.7. 1950

In Kraft: 29. 7. 1950

Dazu Regulations Nr. 21 von 1951

Amtlicher Text: Commonwealth Acts 1950, S. 2734

Englischer Text: Parry, S. 603

LCN, S. 28

14. Nationality and Citizenship Act, Nr. 58

14. 12.1950

In Kraft: 14. 12. 1950

Ändert Sect. 5, 14, 15, 36

Englischer Text: State Papers, Bd. 157, S. 1

LCN, S. 29

15. Nationality and Citizenship Act, Nr. 70

1. 11.1952

In Kraft: 29. 11. 1952

Ändert Sect. 15, 16, 36, 48

Amtlicher Text: Acts 1952, S. 258

Englischer Text: LCN, S. 30

16. Nationality and Citizenship Act, Nr. 85

11. 12. 1953

In Kraft: 8. 1. 1954

Ändert Sect. 5, 12, 14, 15, 16, 25, 32, 41, 53, Schedule 2

Amtlicher Text: Acts 1953, S. 305

Englischer Text: LCN, Nachtrag S. 3

17. Nationality and Citizenship Act, Nr. 1

In Kraft: 11. 5. 1955, aber Sect. 8 (1): 26. 1. 1949

11. 5. 1955

Andert Sect. 7, 12, 14, 15, 25, 29, 36, 37, 46, hebt auf Sect. 31, fügt ein 40 A

Englischer Text: LCN, Nachtrag S. 5 
18. Cocos (Keeling) Islands Act, Nr. 34

In Kraft: 23. 11. 1955 (Gazette 1955, S. 3665). Dazu Regulations vom 23. 11. 1955 (s. u.)

StAB: Sect. 4, 14-15

19. Christmas Islands Act, Nr. 41

2. 9.1958

In Kraft: 2. 9. 1958 (Sect. 1-4) bzw. 1. 10. 1958 (Rest) (Gazette, S. 3185)

StAB: Sect. 15-16

Amtlicher Text: Acts 1958, S. 486

20. Nationality and Citizenship Act, Nr. 63

8. 10.1958

In Kraft: 8. 10. 1958, außer Sect. 9 (1. 6. 1959)

Ändert Sect. 4, 5, 7, 21, 22, 25 (7), 30, 50; hebt auf Sect. 20; fügt ein $23 \mathrm{~A}-\mathrm{B}$

Amtlicher Text: Acts 1958, S. 608

21. Nationality and Citizenship Act, Nr. 79

1. 12.1959

In Kraft: 1. 12. 1959

Ändert Sect. 7, 11, 15, 42, 45, 46, 47, fügt ein 46 A

Amtlicher Text: Acts 1959, S. 356

22. Nationality and Citizenship Act, Nr. 82

13. 12.1960

In Kraft: 13. 12. 1960

Andert Sect. 36

Amtlicher Text: Acts 1960, S. 403

23. Nationality and Citizenship Act, Nr. 11

6.5. 1966

In Kraft: 6. 5. 1966

Ändert Sect. 5, 10, 11, 16, 25, 26, 34, Schedule 2; fügt Schedule 3 hinzu; ändert Währung in Sect. 48, 49, 50, 53

24. Nationality and Citizenship Act, Nr. 11

26.4. 1967

In Kraft: 24. 5. 1967

Ändert Sect. 5, 12, 15

B. Bundesrecht (Regulations)

1. (Erste) Citizenship Regulations, Nr. 4

20. 1.1949

Amtlicher Text: Gazette von 25. 1. 1949

2. Citizenship Regulations Amendment, Nr. 46

15. 6. 1951

Andert Regulations 3, 9, 11, 16, Schedule 1 und 2

Amtlicher Text: Gazette vom 18. 6. 1951

3. Citizenship Regulations Amendment, Nr. 86

8. 8.1951

Fügt ein Regulation $11 \mathrm{~A}$

Amtlicher Text: Gazette vom 9. 8. 1951

4. Citizenship Regulations Amendment, Nr. 120

12. 10.1951

Andert Regulation 3, Schedule 2; fügt ein Regulation 4 A

Amtlicher Text: Gazette vom 15. 10. 1951

5. Citizenship Regulations Amendment, Nr. 24

Ändert Regulations 3, 17, Schedule 1 und 2; hebt auf Regulation $11 \mathrm{~A}$; fügt ein Regulations 21-29

Amtlicher Text: Gazette vom 18. 3. 1954

6. Citizenship Regulations Amendment, Nr. 9

18. 1.1955

Fügt wieder eine Regulation $11 \mathrm{~A}$ ein; ändert Schedule 1 und 2

Amtlicher Text: Gazette vom 27. 1. 1955

7. Citizenship Regulations Amendment, Nr. 80

10. 11.1955

Andert Regulations 3, 8-11, 17, 20, Schedule 1 und 2

Amtlicher Text: Gazette vom 17. 11. 1955

8. Citizenship Regulations Amendment, Nr. 5

7. 2. 1956

Andert Schedule 1

Amtlicher Text: Gazette vom 7. 2. 1956

9. Cocos (Keeling) Islands (Citizenship) Regulations, Nr. 85

Amtlicher Text: Gazette vom 23. 11. 1955 
10. (Zweite) Citizenship Regulations, Nr. 112

21. 12.1956

Amtlicher Text: Gazette vom 24. 12. 1956

11. Citizenship Regulations Amendment, Nr. 23

19. 3. 1959

Ändert Regulations 3, 5, 7, 8, 14, 15, 17, 18, 21, Schedule 2 (neu J A)

Amtlicher Text: Gazette vom 2. 4. 1959

12. Citizenship Regulations Amendment, Nr. 27

18. 4. 1959

Andert Regulations 21

Amtlicher Text: Gazette vom 23. 4. 1959

13. Citizenship Regulations Amendment, Nr. 36

21. 5.1959

Andert Regulations 3, 22-29, Schedule 2 (M)

Amtlicher Text: Gazette vom 28. 5. 1959

14. Citizenship Regulations Amendment, Nr. 78

14. 9. 1959

Fügt ein Regulation $5 \mathrm{~A}$

Amtlicher Text: Gazette vom 24. 9. 1959

15. (Dritte) Citizenship Regulations, Nr. 62

August 1960

Amtlicher Text: Gazette vom 18. 8. 1960

Englischer Text: Exemplar der Forschungsstelle (in der Fassung von 1966)

16. Citizenship Regulations Amendment, Nr. 120

Fügt ein Regulation $5 \mathrm{~A}$; ändert Regulation 25

Amtlicher Text: Gazette vom 5. 10. 1961

17. Citizenship Regulations Amendment, Nr. 1

Januar 1964

Ändert Regulations 5 A, 7; fügt ein Regulations 7 A, 7 B, 7 C, 7 D; ändert Schedule 1 (D-I)

Amtlicher Text: Gazette vom 16. 1. 1964

18. Citizenship Regulations Amendment, Nr. 8

Januar 1965

Ändert Regulation $5 \mathrm{~A}$; hebt auf Regulation 21 und Schedule 3

Amtlicher Text: Gazette vom 18. 1. 1965

19. Citizenship Regulations Amendment, Nr. 146

Oktober 1965

Andert Regulations $5 \mathrm{~A}, 7$

Amtlicher Text: Gazette vom 14. 10. 1965

C. Gliedstaaten

I. NEUSÜDW ALES

1. Gesetz Nr. 6 betr. "letters of denization“

Englischer Text: Oliver's Statutes (1879), Bd. 1, S. 664

2. Gesetz Nr. 39 betr. Ausländer

Ubernimmt den brit. Aliens Act von 1844 (7/8 Vict. c. 66) weitgehend

Englischer Text: Carey's Statutes of NSW (1871), Bd. 1, S. 18

3. Gesetz Nr. 8 betr. Ergänzung des Ausländergesetzes (Eid)

Englischer Text: Carey's Statutes of NSW (1871), Bd. 1, S. 21

4. Gesetz Nr. 19: Naturalization Act

Ubernimmt weitgehend das britische Einbürgerungsgesetz von 1870; hebt auf Akte von 1848 und 1853

5. Naturalization and Denization Act, Nr. 21

27. 7.1898

Hebt auf Akte von 1828 und 1875

Englischer Text: Public Acts of NSW 1957, Bd. 8, S. 215

State Papers, Bd. 90, S. 1239

II. QUEENSLAND

1. Gesetz Nr. 9 betreffend Ausländer

1861

Ändert das NSW-Gesetz von 1848 und hebt das von 1853 auf

2. Gesetz Nr. 3 betreffend Verbot der Einbürgerung von Chinesen 
3. Gesetz Nr. 28: Aliens Act (Consolidation des Gesetzes von 1861) 28.12.1867 Dadurch trat keine Textänderung ein. Erstreckt auf Papua durch Ordinance Nr. 1 von 1889

In Kraft: 31. 12. 1867

Amtlicher Text: Public Acts of Queensland 1936, Bd. 1, S. 356

Englischer Text: State Papers, Bd. 69, S. 456

\section{SÜDAUSTRALIEN}

1. Ordinances betr. Einzeleinbürgerungen

3 Vict. Nr. 4 (1839)

$1839-1847$

3 Vict. Nr. 9 (1840), abgelehnt

5 Vict. Nr. 6 (1841)

1845, Nr. 12

1847, Nr. 4

Alle aufgehoben durch State Law Revision Act Nr. 2168 von 1934

2. Ordinance Nr. 11 betr. Denization

Abgelehnt

3. Ordinance Nr. 7 betr. Annahme des britischen Gesetzes von 1844

Aufgehoben durch das Gesetz von 1858

4. Ordinance Nr. 1 betr. Änderung der Ordinance von 1846

Aufgehoben durch State Law Revision Act Nr. 2168 von 1934

5. Ordinance Nr. 8, betr. Änderung der Ordinance von 1846

Aufgehoben durch das Gesetz von 1858

6. Einbürgerungsgesetz Nr. 20 von $1857 / 58$

$1855 / 56$

Ersetzt den Akt von 1846. Aufgehoben durch das Gesetz von 1864

1858

7. Gesetz Nr. 20 betr. Anerkennung von britischen und australischen Einbürgerungen Aufgehoben durch das Gesetz von 1864

1860

8. Gesetz Nr. 26, Aliens Act: betr. Anerkennung von britischen Einbürgerungen

Abgelehnt (s. Parry, S. 525, Anm. 19)

9. Einbürgerungsgesetz, Nr. 5 (Amending and Consolidating)

Aufgehoben durch Eigentumsgesetz 2328 von 1936, Sect. 4 m. Schedule

Englischer Text: State Papers, Bd. 70, S. 722

10. Gesetz Nr. 5 betr. Änderung des Einbürgerungsgesetzes (Gebühren) Aufgehoben durch das Gesetz von 1895

11. Gesetz Nr. 489 betr. Änderung des Einbürgerungsgesetzes Aufgehoben durch State Law Revision Act 1934, Nr. 2168 Englischer Text: State Papers, Bd. 82, S. 1074

12. Gesetz Nr. 616 betr. Änderung des Einbürgerungsgesetzes Hebt auf Sect. 16 und den Act von 1872; macht Einbürgerung gebührenfrei. Aufgehoben durch das Gesetz von 1934

Englischer Text: State Papers, Bd. 88, S. 1049

\section{TASMANIEN}

1. Act of Council Nr. 4 v. Van Diemen's Land betr. Letters of Denization 1834 Aufgehoben durch das Gesetz von 1861

2. Aliens Act, Nr. 2

30. 11.1861

Ersetzt das Gesetz von 1834. Aufgehoben durch Aliens Act Nr. 12 von 1913 (enthält selber keine StAB)

Englischer Text: State Papers, Bd. 69, S. 684

\section{VICTORIA}

1. Gesetz Nr. 166 betr. Ausländer

Ersetzt das NSW-Gesetz von 1848, das in Victoria galt 
2. Gesetz Nr. 256 betr. Ausländer („The Aliens' Statute“)

i. K. 1. 6. 1865

Ersetzt das Gesetz von 1863, indem es zwei beanstandete Passagen ändert

3. Gesetz Nr. 1063: Aliens Act, betr. Einbürgerung

10. 7.1890

Englischer Text: State Papers, Bd. 82, S. 1075

4. Gesetz Nr. 1462: Aliens Act Amendment

24. 12.1896

Ändert Sect. 5, 7, 8, 10

Englischer Text: State Papers, Bd. 88, S. 1048

VI. WESTAUSTRALIEN

1. Gesetze betr. Einbürgerung von 3 Personen

$4 / 5$ Vict. Nr. 5 (Preiss)

$4 / 5$ Vict. Nr. 6 (Waldeck)

$4 / 5$ Vict. Nr. 12 (Simmons)

2. Gesetz betr. Einbürgerung von Helmich

6 Vict. Nr. 12

3. Gesetz betr. Bestätigung der Einbürgerungen von 1841 12 Vict. Nr. 9

4. Gesetze betr. Einzeleinbürgerungen

$1849-1871$

(Liste: Parry, S. 526, Anm. 16)

5. Einbürgerungsgesetz, Nr. 2

\section{VERTRÄGE}

A. Multilaterale Verträge

Hierbei handelt es sich in allen Fällen um selbständige Beteiligung Australiens, das neben Großbritannien als Partner auftrat.

1. Haager Konvention vom 12. 4. 1930: Australien hinterlegte am 10. 11. 1937 seine Urkunde, und zwar mit dem Vorbehalt, daß das Abkommen hinsichtlich seiner Außengebiete nur für Papua und Norfolk, nicht für Nauru und Neuguinea gelte.

2. Die 3 Haager Protokolle vom 12. 4. 1930: Australien hinterlegte am 8. 7. 1935 ohne Vorbehalte für Außengebiete.

3. UN-Konvention über die StA der Ehefrau vom 29. 1. 1957: Australien trat am 14. 3. 1961 bei, wirksam für sämtliche Außengebiete.

B. Bilaterale Verträge

1. Notenwechsel betr. Austausch von Einbürgerungsmitteilungen mit Deutschland vom 19. 9. 1950: Australien wünschte vierteljährliche Unterrichtung über Einbürgerungen in Deutschland; Deutschland stimmte zu, Gegenseitigkeit wurde gewährt. Der Text ist unveröffentlicht. Näheres: Mitteilung des BMI an die Innenminister vom 13. 3. 19511412 A - 779 III/50, in: GMBl. 1951, S. 85-86 mit Muster.

\section{Entscheidungen:}

Urteile des Obersten Gerichtshofes in Commonwealth Law Reports:

1. 21. 5. 1920, Jerger v. Pearce: CLR, Bd. 27, S. 526-531

2. 4. 6. 1920, Meyer v. Poynton: CLR, Bd. 27, S. 436-442

3. 6. 6. 1952, Wong Man On v. Commonwealth: CLR, Bd. 86, S. 125-135

Britische Urteile:

C. A. v. 14. 1. 1920: Markwald v. Attorney General: Law Reports (Chancery Division) 1920 I, S. $348-371$ 


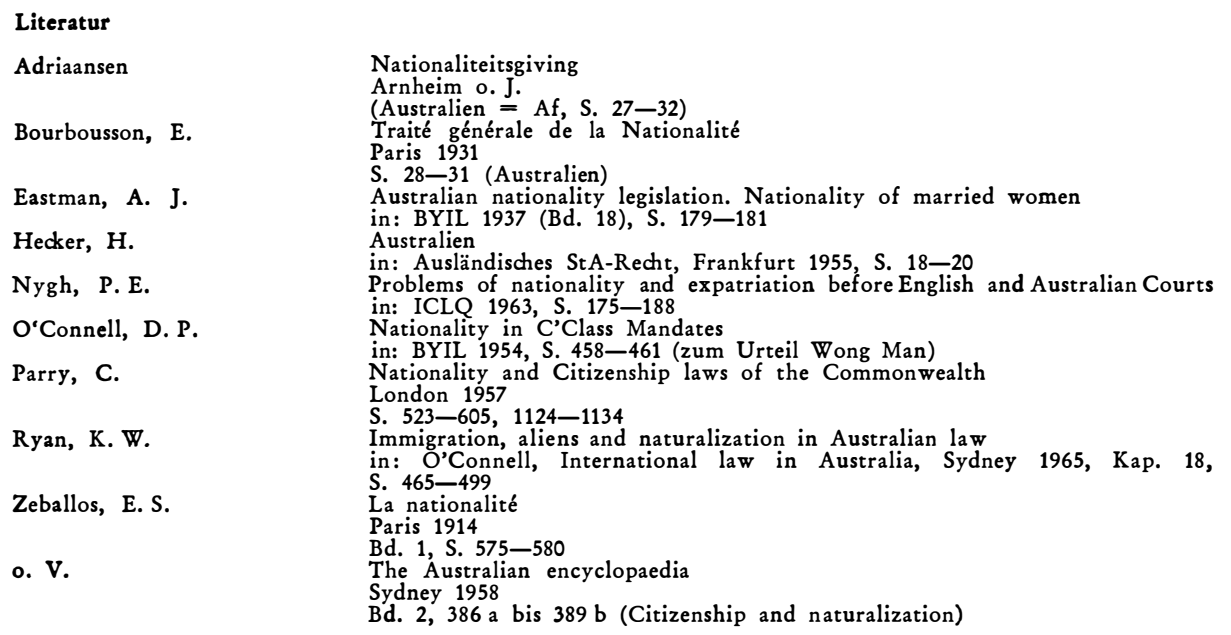

\section{CANTON UND ENDERBURY}

Diese beiden Inseln der Gruppe der Phönix-Inseln, die 1937 den Gilbert- und Ellice-Inseln angegliedert wurden, kamen durch britisch-amerikanisches Abkommen vom 6. 4. 1939 für 50 Jahre unter das Kondominium beider Staaten. Die StA ist darin nicht geregelt. Nach dem britischen StAG von 1948 (Sect. 30 II) konnte dies Gesetz durch Order auf Canton ausgedehnt und diese Insel als Schutzstaat behandelt werden. Das geschah durch Sect. 6 der britischen Protectorats etc. Order von 1949 (= Sect. 7 der neuen Fassung von 1965), die für Canton und Enderbury gilt.

\section{COOK-INSELN}

Britisches Protektorat 1888, Bundesverfassung 1891, 1901 in Neuseeland eingegliedert, 1903 Abtrennung der Nnsel Niue, 1957 gewisse Autonomie. Durch die Verfassung von 1964 eine Art neuseeländischer Schutzstaat mit der britischen Königin als Staatsoberhaupt, aber ohne eigene StA (siehe Killbridge, The Cook Islands Constitution, in NZ University Law Review 1963/65, S. 571-576).

1. Cook Islands Constitution Act Nr. 69

17. 11.1964

Mit Verfassung als Schedule 1

In Kraft: 4. 8. 1965

StAB: Sect. 6 des Act (Einwohner bleiben neuseeländische Staatsangehörige); Sect. 28 der Verfassung (Definition der ständigen Einwohner für Wahlrecht), geändert durch Act Nr. 2 v. 7. 6.1965

Amtlicher Text: Statutes of NZ 1964, S. 459

4. FIJI

Statutes of NZ 1965, S. 46 (Neufassung)

Einheimisches Königreich, seit 1874 britische Kolonie.

1. Aliens Ordinance, Nr. 3

30. 10.1875

Änderungen: 1893, 1923, 1929 und 1945 (s. u.)

Amtlicher Text: Ordinances of the Colony of Fiji 1875-1910, Rev. Ed. 1925, S. 5 (Fassung 1923)

Laws of Fiji, Rev. Ed. 1945, Ch. 54, Bd. 1, S. 651 (Consolidation der Law Rev. 1945)

Englischer Text: State Papers, Bd. 66, S. 648

2. Aliens Amendment Ordinance, Nr. 13

20. 10.1893

Aufgehoben durch Law Revision Ordinance Nr. 9 von 1945

Englischer Text: State Papers, Bd. 85, S. 266 
3. Brit. Nationality and Status of Aliens (Fees) Ordinance, Nr. 16

4. Denaturalisation Ordinance, Nr. 13

5. Law Revision Ordinance, Nr. 22

27. 12.1923

Ändert Sect. 4 von 1875

6. Aliens (Amendment) Ordinance, Nr. 33

18. 10.1929

Ändert Sect. 7, 10; hebt auf 12-13, Schedule C; fügt ein Sect. 9 A

Amtlicher Text: Ordinances 1929, S. 99

7. Brit. Nationality and Status of Aliens (Fees) Ordinance, Nr. 34

18. 11.1929 Amtlicher Text: Ordinances 1929, S. 201 Laws of Fiji, Rev. Ed. 1945, Ch. 56, Bd. 1, S. 663

8. Law Revision Ordinance, Nr. 2

Aindert Sect. 10 (5) von 1875

9. Brit. Nationality (Offences and Fees) Ordinance, Nr. 22

Amtlicher Text: Laws of Fiji, Rev. Ed. 1955, Ch. 66, S. 757

10. Fiji Constitution Order in Council

StAB: Sect. 107 (10) definiert Fijianer und Inder

Amtlicher Text: S. I. 1966, S. 5104

\section{GILBERT- UND ELLICE-INSELN}

Britisches Protektorat 1892, Kolonie seit 12. 1. 1916.

1. Definition (Native) Order, Nr. 3

2. Denaturalisation Order, Nr. 8

Aufgehoben durch Repeal Order Nr. 2 von 1921

3. Native Status Order, Nr. 4

4. Native Status Order, Nr. 2

Amtlicher Text: Laws, Rev. Ed. 1952, c. 17, S. 118

5. Brit. Nationality (Offences and Fees) Ordinance, Nr. 5

Amtlicher Text: Laws, Rev. Ed. 1952, C. 7, S. 28

6. Native Status Amendment Order, Nr. 5

Andert Sect. 2 und 7

7. Order betr. Änderung der Schedule zur Ordinance Nr. 5 von 1949

24. 8. 1951

8. Native Status Amendment Order, Nr. 4

7.7. 1961

Ändert Sect. 6; hebt auf Sect. 5

Amtlicher Text: Orders 1961, S. 42

Western Pacific High Com. Gazette v. 25. 7. 1961, Suppl.

\section{GUAM}

Die spanische Insel wurde im Friedensvertrag vom 10. 10. 1898 an die USA abgetreten, die Staatsangehörigkeit aller abgetretenen Gebiete war in Art. 9 geregelt. Die Insel ist seit 1949 nichtinkorporiertes Territorium der USA.

1. Civil Code of Guam

1. 5.1933

StAB: Sect. 42 a und $42 \mathrm{~b}$

Im neuen Code von 1953 sind diese Vorschriften nicht aufgenommen worden.

Englischer Text: Civil Code, Manila 1933

2. Guam Organic Act (Ch. 512)

1. 8. 1950

StAB: Sect. 4 fügt in das US-StAG von 1940 eine Sect. 206 ein, die dann als Sect. 307 ins StAG von 1952 übernommen wurde.

Amtlicher Text: Statutes at large, Bd. 64, S. 384

Englischer Text: USC 1952, Title 48, \1421 I

Literatur:

Hackworth III, S. $154-155$

Crone, Nr. 36, S. 8-9 


\section{HAWAII}

Das selbständige Königreich Hawaii wurde am 17. 1. 1893 Republik und am 12. 8. 1898 von den USA als Territorium annektiert. Seit 21. 8. 1959 sind die Inseln Staat der USA.

1. Verfassung des Königreichs StAB: Art. 77/8 (Wahlrecht bei Denization)

2. Gesetz betr. Beseitigung von Schwierigkeiten bei der Einbürgerung 28.6.1854 Englischer Text: Civil laws at the Hawaiian Islands, Honolulu 1897, S. 607 (als $₫ 1582-1583$ des Code von 1897)

3. Civil Code für Hawaii

StAB: $\ 429-433$ (das ist Art. 8)

Die weitergeltenden $\$ \int$ wurden in die bereinigten Ausgaben von 1884 und 1897 (s. u.) aufgenommen.

Englischer Text: Martens, N. R. G., 2. Serie, Bd. 19, S. 747

4. Verfassung des Königreichs

20. 8.1864

StAB: über Einbürgerung sowie über Wahlrecht in Art. 62 (geändert 13. 7. 1874)

Amtlicher Text: Laws of Hawaii 1864/65, S. 85

Englischer Text: Thurston, Fundamental law of Hawaii, Honolulu 1904, S. 169 Lydecker, Roster Legislatures of Hawaii, S. 88

5. Einbürgerungsgesetz des Königreichs Hawaii

Amtlicher Text: Sessional laws 1887, Ch. 10

Englischer Text: State Papers, Bd. 78, S. 939

6. Verfassung des Königreichs Hawaii

StAB: Art. 56, 59 und 62

Englischer Text: Foreign Relations 1887, S. 574

7. Zweites Einbürgerungsgesetz

Amtlicher Text: Sessional laws 1890, Ch. 24

8. Verfassung der Republik Hawaii

Inkrafttreten: 4. 4. 1894

StAB: Art. 17-19

Englischer Text: Civil laws, a. a. O., S. 1

State Papers, Bd. 86, S. 312

Hackworth III, S. 125 (nur Art. 17 und 19)

9. Drittes Einbürgerungsgesetz

15. 6.1896

Amtlicher Text: Sessional laws 1896, Act 77

Englischer Text: Civil laws, a. a. O., S. 604 (als $\ 1570-1578$ des Code von 1897)

Foreign Relations 1896, S. 387

10. Bereinigte Ausgabe der Zivilgesetze

1897

StAB: \1570-1587 (Einbürgerung und Ausländer)

Davon sind \1570-1578: Einbürgerungsgesetz von 1896 (s. o.)

1582-1583: Gesetz von 1854 (s. o.)

1580-1581: Einbürgerungsgesetz von 1887 (s. o.)

1579, 1584-1587: Bereinigte Ausgabe 1884, \ 430-432

Amtlicher Text: Civil laws, a. a. O., S. 604-608

11. Hawaiian Organic Act

30. 4.1900

StAB: Sect. 4

Englischer Text: State Papers, Bd. 92, S. 755

12. Immigration and Nationality Act, Nr. 414

Inkrafttreten: 24. 12. 1952

StAB für Hawaii: Sect. 305

27. 6. 1952 
Amtlicher Text: Statutes, Bd. 66, S. 163

Englischer Text: AJ 1953, Suppl. Nr. 2

LCN, 1954, S. 498

Literatur:

Hackworth III, S. $119-127$

Crone, Nr. 35, S. 3-4

\section{NAURU}

Insel, zu Deutsch-Neuguinea 1888, Kondominium Großbritannien, Neuseeland, Australien 1919 (1920 als Mandat, 1947 als Treuhandgebiet), 31. 1. 1968 unabhängig als Republik, jedoch eng mit Australien verbunden.

1. Nauruan Community Ordinance Nr. 2

18. 8.1956

Amtlicher Text: Nauru Gov. Gazette vom 18. 8. 1956

Englischer Text: YHR 1956, S. 265 (Analyse)

Nauruan Community Ordinance 1956-1962, Canberra 1966

2. Nauruan Community Ordinance Nr. 5

7. 12.1962

In Kraft: 31. 12. 1962

Ändert Sect. 5, 9; fügt ein $10 \mathrm{~A}$

Amtlicher Text: Nauru Gov. Gazette v. 12. 12. 1962

3. Constitution of Nauru

29. 1.1968

In Kraft: 31. 1. 1968

StAB: Art. $71-76$

Amtlicher Text: The Constitution of Nauru, o. O., o. J.

4. Constitution Alteration

17. 5.1968

Geänderte StAB: Art. 74; eingefügt eine neue Schedule $5 \mathrm{zu}$ Art. 84, wobei

Punkt VI auf Art. $71-72$ verweist

Englischer Text: Exemplar der FVR

\section{NEUE HEBRIDEN}

Britisch-französisches Kondominium seit Vertrag vom 16. 11. 1887. Die Bewohner dürfen von den beiden Kondominanten weder eingebürgert noch als Schutzgenossen behandelt werden. Nach Sect. 30 II des britischen StAG von 1948 und Sect. 6 der britischen Protectorats etc. Order von 1949 (= Sect. 7 der Order von 1965) gelten die Neuen Hebriden als „Protected State“. Eine Regelung der StA ist gemäß Auskunft des britischen Residenten vom 28. 12. 1967 an die Forschungsstelle für Völkerrecht und ausländisches öffentliches Recht der Universität Hamburg nicht erfolgt.

1. Brit.-franz. Vertragswerk über die Neuen Hebriden

27. 2. / 22. 10. 1906

Inkrafttreten: 2. 12. 1907

StAB: Art. 8 Z. 1-2 (Einbürgerungsverbot der Eingeborenen)

Amtlicher Text: J. O. vom 15. 1. 1907 (Dekret vom 11. 1. 1907)

Englischer Text: State Papers, Bd. 99, S. 229

Englischer und französischer Text: Martens, 3. Serie, Bd. 1, S. 523

Französischer Text: Dareste 1907, S. 161

2. Brit.-franz. Protokoll über die Neuen Hebriden

6. 8. 1914

Inkrafttreten: 8. 3. 1922

StAB: Art. 8 Z. 1-2 (unverändert gegenüber 1906)

Amtlicher Text: LNTS, Bd. 10, S. 335

J. O. vom 4. 6. 1922 (Dekret vom 27. 5. 1922)

Englischer Text: State Papers, Bd. 114, S. 212

Martens, 3. Serie, Bd. 12, S. 198

Dareste 1922, S. 812

10. NEUGUINEA

Deutsche Kolonie 1884, australisches Mandat 1920, seit 1947 Treuhandgebiet.

1. Laws Repeal and Adopting Ordinance, Nr. 1

In Kraft: 9. 5. 1921

6. 5. 1921 
Sect. 11 i. V. mit Schedule 1 Nr. 8: Die dort genannten Gesetze Australiens, u. a. das StAG von 1920 sind anwendbar auf Neuguinea „so far as they are applicable".

Amtlicher Text: Commonwealth Gazette vom 6. 5. 1921

2. Nationality Act (Nr. 2), Nr. 28

13. 8.1946

Sect. 3 fügt neue Sect. $5 \mathrm{~B}$ in das StAG von 1920 ein, womit dies auf das Mandatsgebiet Neuguinea erstreckt wird.

Siehe Australien

3. Nationality and Citizenship Act, Nr. 83

21. 12.1948

Sect. 25 regelt Anwendung für Neuguinea: Dortige British subjects werden Australier.

Siehe Australien

\section{NEUKALEDONIEN}

Französisch seit 24. 9. 1853, besondere Kolonie seit 1860, seit 1946 T. O. M.

1. Dekret betr. Einbürgerung in Neukaledonien

10. 11.1882

Amtlicher Text: J. O. vom 15. 11. 1882

Französischer Text: Clunet 1882, S, 687

State Papers, Bd. 73, S. 68

CNF, Bd. 1, S. 125

2. Dekret betr. Anerkennung der StA Unehelicher in Westafrika, Indien und

Neukaledonien

15. 12.1922

Amtlicher Text: J. O. vom 2. 2. 1923

Französischer Text: Dalloz 1923, S. 83

Dareste 1923, S. 266

3. Dekret betr. Erwerb der StA in Neukaledonien

Amtlicher Text: J. O. vom 5./6. 9. 1932, S. 9728

Französischer Text: Dalloz 1933, S. 212

4. Dekret betr. Einbürgerung alter Kämpfer in West- und Äquatorialafrika,

Indochina, Madagaskar, Neukaledonien und Ozeanien

19. 4.1933

Siehe Polynesien

5. Dekret betr. StA-Erwerb der Mestizen in Neukaledonien

30. 5.1933

Amtlicher Text: J. O. vom 3. 6. 1933, S. 5901

6. Dekrekt betr. französische StA der Eingeborenen in Neukaledonien 19.10.1937 Amtlicher Text: J. O. vom 22. 10. 1937

Französischer Text: CNF, Bd. 1, S. 203

7. Dekret (7) betr. Änderung von Art. 2-7, 13-14 des Dekrets

vom 19. 10. 1937 und Einfügung von Art. 4 bis und 5 bis

Amtlicher Text: J. O. vom 17. 11. 1939

Französischer Text: CNF, Bd. 1, S. 210

8. Dekret (6) betr. Änderung von Art. 6 des Dekrets vom 19. 10. 1937 11. 5. 1940 Amtlicher Text: J. O. vom 19. 5. 1940

Französischer Text: CNF, Bd. 1, S. 215

\section{NEUSEELAND}

Die Konvention über die StA der Ehefrau von 1957 ratifizierte Neuseeland (UNTS,

Bd. 317, S. 380), gültig für Cook, Tokelau, Samoa.

1. Kgl. Instruktionen an Gouverneur Hobson

5. 12.1840

StAB : $\int 17$

Amtlicher Text: Ordinances of NZ 1848-49, Wellington 1850, Bd. 9, S. 14

2. Ordinances (NZ) betreffend Einbürgerung von Deutschen

$1844-1848$

1844: 8 Vict. Sess. 3, Nr. 2 (53 Deutsche)

1845: 8 Vict. Sess. 5, Nr. 1-2

1846: 10 Vict. Sess. 7, Nr. 1 
1847: 11 Vict. Sess. 8, Nr. 14

1848: 12 Vict. Sess. 9, Nr. 4

3. Ordinance der Provinz Munster betreffend Einbürgerung zweier Deutscher und eines Italieners

13 Vict. Sess. 1, Nr. 1

4. Ordinance (NZ) betreffend Einbürgerung verschiedener Personen

15 Vict. Sess. 11, Nr. 9

5. Ordinances betreffend Einbürgerungsbestätigungen

$1853-1865$

16 Vict. Sess. 12, Nr. 4

18 Vict. Sess. 1, Nr. 2

19 Vict. Sess. 3, Nr. 1

$19 / 20$ Vict. Sess. 1 , Nr. 2

21/22 Vict. Sess. 2, Nr. 48

24 Vict. Nr. 31

25 Vict. Nr. 7

26 Vict. Nr. 25

27 Vict. Nr. 36

28 Vict. Nr. 12

29 Vict. Nr. 67

6. Native Rights Act, Nr. 11

1865

StAB: Sect. 2

7. Aliens Act, Nr. 17

8. Act Nr. 61 betreffend verschiedene Einbürgerungen

4. 10.1866

1866

1866

1869

9. Act Nr. 76 betreffend Bestätigung einer Einbürgerung von 1866

11. Aliens Act, Nr. 40

12. 9.1870

12. Naturalization Act 1870 (Fees) Act Nr. 47

14. 11.1871

Englischer Text: State Papers, Bd. 65, S. 1215

17. 8.1880

13. Aliens Act, Nr. 10

Englischer Text: State Papers, Bd. 71, S. 238

US Report vom 20. 12. 1906, S. 423

14. Children of Naturalized Persons (Charge for Letters of Naturalization)

Act Nr. 17

Englischer Text: State Papers, Bd. 73, S. 604

15. Aliens Act (Amendment) Act, Nr. 19

13. 4. 1882

Englischer Text: State Papers, Bd. 85, S. 319

24. 9.1892

US Report vom 20.12.1906, S. 426

16. Asiatic Restriction Act

1896

1908

17. Aliens Act Nr. 5

15. 9.1917

18. Revocation of Naturalization Act, Nr. 8

9. 8.1920

20. Finance Act 1921/22, Nr. 72

4. 2. 1921

StAB: Sect. 9

21. Brit. Nationality and Status of Aliens (in New Zealand) Act Nr. 46 von 1923

20. 2. 1924

In Kraft: 13. 5. 1924

Englischer Text: State Papers, Bd. 121, S. 567

22. Brit. Nationality and Status of Aliens (in New Zealand) Amendment Act, Nr. 62

6. 11.1924

Ändert Sect. 9; schränkt ein Sect. 5 (1, b) f. Samoa

Englischer Text: State Papers, Bd. 121, S. 567

23. Brit. Nationality and Status of Aliens (in New Zealand) Act, Nr. 58 von 1928

In Kraft: 1. 7. 1929 (Gazette, S. 1314) 
Gebilligt durch kgl. Order vom 29. 1. 1929 (State Papers, Bd. 130, S. 12)

Sect. 7-8 gelten für Samoa

Amtlicher Text: Public Acts 1908-1931, Bd. 1, S. 159

Englischer Text: Flournoy, S. 104

State Papers, Bd. 130, S. 82

Parry, S. 612 (ohne Sect. 9-14)

Französische Úbersetzung: Bourbousson, S. 503

24. Brit. Nationality and Status of Aliens (in NZ) Regulations

Amtlicher Text: Gazette NZ 1929, S. 2418

25. Brit. Nationality and Status of Aliens (in NZ) Amendment Act Nr. 38 von 1934/35

Paßt das brit. StA-Änderungsgesetz von 1933 für Neuseeland an

Amtlicher Text: Statutes 1934/35, S. 381

26. Brit. Nationality and Status of Aliens (in New Zealand) Amendment Act, Nr. 13

Paßt das britische StA-Änderungsgesetz von 1943 für Neuseeland an

Amtlicher Text: Statutes 1943, S. 59

Englischer Text: Parry, S. 616

27. Brit. Nationality and Status of Aliens (in New Zealand) Amendment Act, Nr. 20

Betrifft StA der Frau; hebt auf das Gesetz von 1934/35 und Sect. 10 des britischen StAG (für Neuseeland)

In Kraft: 9. 10. 1946

Amtlicher Text: Statutes 1946, S. 185

Englischer Text: Parry, S. 618

28. British Nationality and New Zealand Citizenship Act, Nr. 15

6. 9.1948

In Kraft: 1. 1. 1949

Änderungen dieses StAG: 1959, 1961, 1962, 1965

Ferner werden Verweise in der 3. Schedule (Änderung anderer Gesetze) auf 2 Gesetze nach deren Aufhebung aufgehoben: Nr. 3 vom 18. 8. 1950 hebt auf Parlamentsgesetz von 1908 (Statutes 1950, S. 8), Nr. 76 vom 1. 10. 1954 hebt auf Gemeindeverbandsgesetz von 1933 (Statutes 1954, S. 954). Durch Republic of Ireland Act Nr. 13 vom 4. 9. 1950 gelten Hinweise auf Irland als solche auf die Republik Irland (Statutes 1950, S. 41).

Amtlicher Text: Statutes 1948, S. 144

Statutes Repr. 1908-1957, Bd. 1, S. 657

Deutsche Übersetzung: Bergmann, 16. Lief. vom 30. 8. 1958, Neuseeland, S. 1

Englischer Text: LCN, S. 337

State Papers, Bd. 151, S. 769

Peaslee, 2. Aufl., Bd. 2, S. 866

Bettoni, Bd. 2, S. 328

Adriaansen, Nc, S. 1

Parry, S. 624

29. Aliens Act, Nr. 28

12. 11.1948

StAB: Sect. 20 (Beweislast) und 21 (Aufhebungen)

In Kraft: 1. 1. 1949

Durch dieses Gesetz (Sect. 21 a) wird Sect. 13 des StAG von 1928, die durch das StAG von 1948 aufrechterhalten worden war, aufgehoben.

Amtlicher Text: Statutes 1948, S. 249

Englischer Text: State Papers, Bd. 151, S. 793

30. Brit. Nationality and NZ Citizenship Regulations Nr. 7

31. Registration of Birth and Death (Citizenship) Regulations Nr. $93 \quad 1949$

32. Citizenship Deprivation Rules Nr. 121

1949

33. Brit. Nationality and NZ Citizenship Amendment Regulations Nr. 154

1949 
34. Infants Act (Amendment) Act Nr. 18

4. 9.1950

StAB: Sect. 2 schafft neue Sect. 21, darin nach Subsection 2 e kein StA-Erwerb

durch Adoption

Amtlicher Text: Statutes 1950, S. 50

Englischer Text: LCN, S. 351

35. Adoption Act, Nr. 93

27. 10. 1955

StAB: Sect. 16 (2 e), keine StA durch Adoption

Amtlicher Text: Statutes 1955, S. 1128

36. Samoa Amendment Act Nr. 22

StAB: Sect. 32 und Schedule 3 hebt auf Sect. 33 (4) des StAG von 1948

Amtlicher Text: Statutes 1957, S. 181

37. Brit. Nationality and N.Z. Citizenship (Amendment) Act, Nr. 38 15. 10. 1959

In Kraft: 15. 10. 1959

Aindert Sect. 3, 7, 8, 9, 12, 23, 26; fügt ein 9 A, 32 A

Amtlicher Text: Statutes 1959, S. 344

Deutsche Úbersetzung: Bergmann, 31. Lief. vom 1. 10. 1965 (Gesetz von 1948 i. d. F. von 1959)

38. Brit. Nationality and NZ Citizenship Regulations Nr. 64

18. 5.1960

Ersetzt die Regulations von 1949, Nr. 7

Amtlicher Text: Gazette vom 19. 5. 1960

39. Registration of Births and Deaths (Citizenship) Regulations Nr. 91 22.6. 1960 Ersetzt die Regulations von 1949, Nr. 93

Amtlicher Text: Gazette vom 23. 6. 1960

40. Western Samoa Act, Nr. 68

24. 11.1961

In Kraft: 1. 1. 1962

StAB: Sect. 10 (4 d); Schedule 2 ändert StAG, Sect. 2 (1), 5 (2), 8 (1), 12 (1), 21 (1); Schedule 3 hebt auf Sect. 33 (1, c-d) des StAG sowie Samoa Act von 1929; Sect. 6 fügt eine Sect. 5 A in den Aliens Act von 1948 ein, so daß Samoaner in NZ nicht registrierpflichtig sind.

Amtlicher Text: Statutes 1961, S. 1584

41./42. Brit. Nationality and N. Z. Citizenship Orders

1961 und 1962

43. Brit. Nationality and N. Z. Citizenship (Amendment) Act, Nr. 26 23.11. 1962 Ändert Sect. 3 und 8. Betrifft Südafrika

Amtlicher Text: Statutes 1962, S. 216

44./45. Brit. Nationality and N.Z. Citizenship Orders

1963 und 1964

46. Brit. Nationality and N. Z. Citizenship (Amendment) Act, Nr. 72 27.10. 1965 Andert Sect. 3, 7, 31; hebt auf Sect. 3 des Act Nr. 38 von 1959

Amtlicher Text: Statutes 1965, S. 750

47. Brit. Nationality and NZ Citizenship Regulations (Amendment Nr. 1),

Nr. 117

Ändert Regulation 11

7.6. 1967

Amtlicher Text: Gazette vom 8.6.1967

48. Brit. Nationality and NZ Citizenship Order Nr. 73

17. 4. 1967

Fügt Guayana zu den Commonwealth-Ländern in Sect. 3 (3) des StAG von 1948 hinzu

Amtlicher Text: Gazette vom 20. 4. 1967

Entscheidungen:

Urteile in New Zealand Law Reports:

1. 6. 3. 1884 , Hunt v. Gordon, NZLR 1884 I, S. 160-244

2. 25. 9. 1917, Maseman v. Maseman, NZLR 1917, S. 769-774

3. 18. 2. 1919, in re Horne, NZLR 1919, S. 190-193

4. 8. 7. 1919, Lum v. Attorney General, NZLR 1919, S. 741-753

5. 28. 9. 1922, in re Wolter, NZLR 1923, S. 328-336 


\section{Literatur}

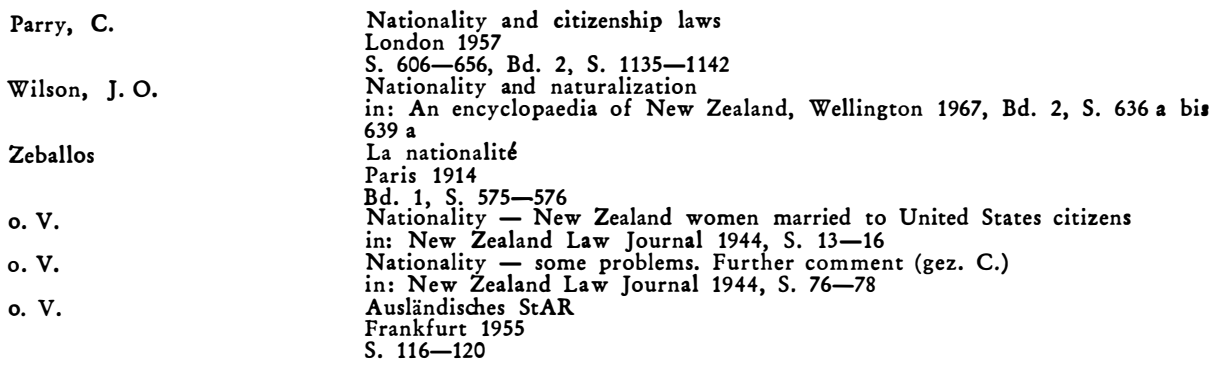

13. NIUE

Britisches Protektorat 1900, 1901 in Neuseeland eingegliedert und Cook unterstellt, 1903 davon abgetrennt. 1957 gewisse Autonomie, 1966 erweitert. Das neuseeländische StAG gilt dort.

\section{PAPUA}

1884 britisches Protektorat Britisch-Neuguinea, 1888 britische Kolonie, 1902 australisch, seit 1906 als Papua-Territorium.

1. Ordinance, Nr. 1

Erstreckt das Ausländergesetz Queenslands von 1867 auf Papua

\section{Aliens Ordinance, Nr. 26}

6. 10.1911

Ersetzt das Gesetz von 1867

In Kraft: 6. 3. 1912 (Veröffentlichung des assent in Government Gazette)

Amtlicher Text: Statute Law of Papua, Bd. 2, 1919, S. 14

Englischer Text: State Papers, Bd. 104, S. 547

3. Ordinance Revision Act Nr. 3 von 1913

Ändert Sect. 12 der Ordinance von 1911 (Fees)

20. 1.1914

Amtlicher Text: Statute Law of Papua, Bd. 2, 1919, S. 736

4. Australian Nationality Act, Nr. 24

StAB für Papua: Sect. 5 A eingefügt, erstreckt das australische StAG von 1920 auf Papua

Texte: siehe Australien

5. Ordinance, Nr. 15

30. 12. 1922

Hebt die Ordinance von 1911 auf, nachdem das australische StAG auf Papua erstreckt worden war

\section{PAZIFISCHE INSELN VON MIKRONESIEN}

Die Karolinen und Marianen waren spanisch, wurden 1899 deutsch (die MarschallInseln waren es schon seit 1885), 1914 von Japan besetzt und ab 1920 dessen Mandat. Seit 1947 sind die Inseln Treuhandgebiet der USA.

1. Treuhandabkommen mit den USA

StAB: Art. 11

In Kraft: 18. 7. 1947

Amtlicher Text: UNTS, Bd. 8, S. 189

Englischer Text: State Papers, Bd. 148, S. 513

Französischer Text: NE, Nr. 545

2. Code des Treuhandgebietes

22. 12.1952

StAB: Ch. 10, Sect. 660-662

Amtlicher Text: Code of the T'rust Territory of the Pacific Islands, Rev. Ed., Saipan 1966, S. 197

3. Executive Order Nr. 97

Betrifft Anderung von Chapter 10 und Sect. 875

In Kraft: 1. 3. 1964 


\section{6. (FRANZOOSISCH)-POLYNESIEN}

Tahiti war französisches Protektorat ab 1843. 1880 wurde es annektiert. 1885 wurde die Kolonie der "Französischen Niederlassungen in Ozeanien“ gegründet, sie trägt als T. O. M. seit Gesetz vom 26. 7. 1953 den Namen „Französisch-Polynesien".

1. Gesetz betr. Ratifikation der Erklärungen vom 29. 6. 1880 über die Annexion von Tahiti durch Frankreich

StAB: Art. $3-4$

Amtlicher Text: J. O. vom 1. 1. 1881

Französischer Text: State Papers, Bd. 71, S. 220

Dalloz 1882, S. 10

CNF, Bd. 2, Suppl., S. IV

2. Dekret betr. Anerkennung Unehelicher in Ozeanien

24. 4. 1919

Amtlicher Text: J. O. vom 2. 5. 1919

Französischer Text: Niboyet-Goulé, 1929, S. 86

3. Dekret (3) betr. Erwerb der französischen StA in Ozeanien

8. 11.1921

Amtlicher Text: J. O. vom 13. 11. 1921

Französischer Text: State Papers, Bd. 114, S. 753

Dareste 1922, S. 268

4. Gesetz betr. französische Einbürgerung in Ozeanien

6. 12.1932

Amtlicher Text: J. O. vom 10. 12. 1932

Französischer Text: State Papers, Bd. 135, S. 495

Bourbousson, Anhang 1938, S. 39

5. Dekret betr. Einbürgerung eingeborener alter Kämpfer in ... Ozeanien

Amtlicher Text: J. O. vom 25. 4. 1933, S. 4324

19. 4. 1933

Deutsche Ubersetzung: SGS, Bd. 28, S. 58

Französischer Text: State Papers, Bd. 136, S. 668

Bourbousson, Anhang 1938, S. 25

CNF, Bd. 2, Suppl., S. IV

6. Dekret betr. französische StA in Ozeanien

Amtlicher Text: J. O. vom 18. 7. 1933

Französischer Text: Bourbousson, Anhang 1938, S. 42

State Papers, Bd. 136, S. 672

CNF, Bd. 1, S. 190

7. Dekret (6) betr. Anderung von Art. 2-7, 13-14 des Dekretes vom 9. 7.1933

und Einfügung von Art. 4 bis und 5 bis

Amtlicher Text: J. O. vom 17. 11. 1939

12. 11.1939

Französischer Text: CNF, Bd. 1, S. 197

8. Dekret (5) betr. Änderung von Art. 6 des Dekrets vom 9. 7. 1933 11.5.1940

Amtlicher Text: J. O. vom 19. 5. 1940

Französischer Text: CNF, Bd. 1, S. 202

\section{SAMOA (OST)}

Nach einem deutsch-britisch-amerikanischen Kondominium über ganz Samoa von 1889-1899 wurden die östlichen Inseln 1899 an die USA abgetreten, die die Inseln aber erst 1921 annektierten, jedoch nicht als direkte Besitzung, sondern als "outlying possession". Die Angehörigen solcher Gebiete galten nach dem StAG von 1940 als "nationals without citizenship" (Sect. 204 i. V. m. 101 b und e). Nach dem StAG von 1952 waren nur noch Samoa und Swains Islands solche "outlying possessions" (Sect. 101, Ziff. 29), die frühere Sect. 204 wurde jetzt Sect. 308.

Literatur:

Hackworth III, S. $155-157$

Crone, Nr. 36, S. 9 


\section{SAMOA (WEST)}

Deutsch 1899-1914, Neuseeländisches Mandat 1920, unabhängig von Neuseeland am 1. 1. 1962. Vertrag betr. Úbernahme von Staatsverträgen vom 30. 11. 1962 (UNTS, Bd. 476, S. 3). Die Konvention über die StA der Ehefrau von 1957 war von Neuseeland auf Samoa erstreckt worden (UNTS, Bd. 317, S. 380).

1. Bekanntmachung des Gouverneurs von Samoa betr. Auslegung des Begriffs Eingeborener

Deutscher Text: Kol. ges. geb., Bd. 5, S. 104

2. Samoa Act, Nr. 16

StAB: Sect. 3 definiert „Samoan“

Englischer Text: State Papers, Bd. 121, S. 500

3. Samoa Act Amendment Act, Nr. 25

StAB: Sect. 2, ändert Sect. 3 "Samoan“

Amtlicher Text: Public Acts 1908-1931, S. 874

4. Brit. Nationality and Status of Aliens (in New Zealand) Act, Nr. 58 von 1928

StAB für Samoa: Sect. $7-8$

29. 1.1929

Texte: siehe Neuseeland

5. Western Samoa (New Zealand Protected Persons) Order Nr. 158

Aufgehoben durch Western Samoa Act, Nr. 68 von 1961

(Siehe Neuseeland)

6. Samoa Act Amendment Act Nr. 74

6. 12. 1951

Ersetzt den Ausdruck „Eingeborene" überall durch "Samoaner"

Amtlicher Text: Statutes NZ 1951, S. 479

7. Citizenship of Western Samoa Ordinance Nr. 11

Amtlicher Text: (engl. und samoanisch) Ausgabe der Ordinance, Apia 1959, $28 \mathrm{~S}$.

Englischer Text: YHR 1959, S. 339 (Auszug)

8. Western Samoa Act, Nr. 68

Siehe Neuseeland

24. 11.1961

\section{9. (BRITISCH-)SOLOMON-INSELN}

Britisches Protektorat seit 1893, dem Oberkommissar des Westpazifiks unterstellt. Nach der British Protectorates etc. Order vom 29. 10. 1965 sind die Bewohner weiterhin staatsangehörigkeitsrechtlich Schutzgenossen.

1. High Com. of Western Pacific Brit. Nationality Offences and Fees Order 1951

2. Immigration Ordinance, Nr. 8

16. 8.1955

In Kraft: 1. 1. 1956

StAB: Sect. 3

Amtlicher Text: Laws, Rev. Ed. 1961, c. 21, S. 278

\section{TOKELAU}

Die Union-Inseln 1877 britisches Protektorat, 1916 annektiert und Gilbert/ElliceInseln unterstellt, $1925 \mathrm{zu}$ Neuseeland als Dependenz. 1946 Name in Tokelau geändert. Die Einwohner sind Neuseeländer und besitzen seit 1948 eine geringe Eigenverwaltung.

\section{TONGA}

Königreich, seit 1900 unter britischem Protektorat. Die Konvention über die StA der Frau vom 29. 1. 1957 wurde durch Notiz vom 3. 11. 1960 auf Tonga erstreckt (UNTS, Bd. 379, S. 438), dagegen nicht die Staatenlosen-Konvention vom 28. 9. 1954. 
StAB: Sect. 29 (Sect. 32 in Ausgabe von 1903)

Amtlicher Text: Law of Tonga, Rev. Ed. 1929, S. 680 (Fassung 1928)

Englischer Text: State Papers, Bd. 135, S. 912

2. Law of Adoption, Nr. 10

StAB: Sect. 3 und 5 (StA-Erwerb durch Adoption)

3. Constitution (Amendment) Act, Nr. 35

16. 7.1906

Ändert Sect. 32 der Verfassung (Einbürgerung)

4. Naturalisation Act, Nr. 6

Amtlicher Text: Law of Tonga, Rev. Ed. 1929, Ch. 24, S. 290

Englischer Text: Parry, S. 364

5. Maintenance of Illegitimate Children Act, Nr. 19

StAB: Sect. 17-18 (StA-Erwerb durch Adoption)

Amtlicher Text: Law of Tonga, Rev. Ed. 1929, Ch. 22, S. 281

6. Tongan Nationality Act, Nr. 11

Englischer Text: State Papers, Bd. 139, S. 160

Parry, S. 363

7. Nationality Act (Consolidation)

20. 11.1915

Sect. 2 = Sect. 2 des Nationality Act, 1935

Sect. $3-7=$ Sect. $8-12$ des Naturalisation Act, 1915

Sect. $8-9=$ Sect. 15 und 17 des Naturalisation Act, 1915

Sect. $10-14=$ Sect. $2-5,7$ des Naturalisation Act, 1915

Sect. $15-16=$ Sect. $13-14$ des Naturalisation Act, 1915

Sect. $17=$ Sect. 18 des Naturalisation Act, 1915

Amtlicher Text: Laws of Tonga, Rev. Ed. 1947, Ch. 25

8. Nationality (Amendment) Act, Nr. 7

Ersetzt Part I des Consolidated Act von 1947 (Sect. 2-9), d. h. Sect. 2 von 1935 und Sect. 8-12, 15, 17 von 1915, durch neue Sect. 3-9

Englischer Text: Parry, Bd. 2, S. 1277

\section{WALLIS UND FUTUNA}

Französisches Protektorat seit 1842, T. O. M. seit Volksabstimmung vom 27. 12. 1959.

1. Gesetz Nr. 814 betr. Statut für Wallis und Futuna

29. 7.1961

StAB: Art. 2

Amtlicher Text: J. O. vom 30. 7. 1961, S. 7019 


\section{Quellen-Nachweise}

Das folgende Verzeichnis enthält die Quellen, soweit diese im Text nicht vollständig angegeben sind, ferner einige Abkürzungen. Literatur zum Staatsangehörigkeitsrecht war nur bei Australien und Neuseeland feststellbar: siehe die dort angegebenen besonderen Literaturhinweise.

Adriaansen

AJ

Bergmann

Bettoni

Bourbousson

CLR

Clunet

CNF

Crone

Dalloz

Dareste

Flournoy (und Hudson)

FVR

Hackworth

J. 0 .

Kol.ges.geb.

LCN

LNTS

Martens

$\mathrm{NE}$

Niboyet-Goulé

NSW

NZ

Parry

Peaslee

StAB

StAG

State Papers

SGS

S. I.

UNTS

USC

Verfassungsregister

YHR
Nationaliteitswetgiving

Arnheim o. J. (Loseblattsammlung)

American Journal of International Law

Internationales Ehe- und Kindschaftsrecht

3. Aufl., Frankfurt/M. (Loseblattsammlung)

L'italiano nel mondo .

2 Bände, Rom 1954 und 1955

Traité général de la nationalité

Paris 1931 (Nachtrag 1938)

Commonwealth Law Reports (Australien)

Journal de Droit international privé

Code de la Nationalité Française

2 Bände, Melun 1945

Citizenship status of inhabitants of the territories and outlying possessions

in: Immigration and naturalization service, 2. Serie, Nr. 35 bzw. 36, vom

11. bzw. 18. 2. 1935

Recueil périodique..

(Paris)

Recueil de législation et jurisprudence coloniales

(Paris)

A collection of nationality laws

New York 1929

Forschungsstelle für Völkerrecht

Digest of international law

Bd. 3, New York 1942

Journal Officiel, République Française

(Lois et décrets)

Die deutshe Kolonialgesetzgebung

13 Bände, Berlin 1893-1910

Laws concerning nationality

New York 1954 (Nachtrag 1959)

League of Nations Treaty Series

Nouveau recueil général de traités...

Notes et études, La documentation française

(Paris)

Recueil de textes usuels de droit international

2 Bände, Paris 1929 und 1930 (mit Suppl.)

Neusüdwales

New Zealand

New Zealand Law Reports

Nationality and citizenship laws of the Commonwealth and Ireland

London 1957 (Suppl. 1960)

Constitutions of Nations

3 Auflagen

Staatsangehörigkeits-Bestimmung

Staatsangehörigkeits-Gesetz

British and Foreign State Papers

Sammlung geltender Staatsangehörigkeitsgesetze

herausgegeben von der Forschungsstelle für Völkerrecht

Statutory Instruments (Britische Verordnungssammlung)

United Nations Treaty Series

United States Code

Verfassungsregister Teil IV: Afrika - Asien - Australien

Frankfurt/M. 1963

Yearbook on Human Rights

(New York) 